\title{
Vers un nouveau profil des enseignants et des enseignantes au primaire en Suisse : bref portrait d'un futur dispositif de formation initiale entièrement bilingue
}

\section{HRONIQUE • Point de vue International}

\section{Les raisons de la création du Cursus bilingue}

Nous présentons ici brièvement une nouvelle formation initiale des enseignants et des enseignantes intitulée « Cursus bilingue/ Bilingualer Studiengang». D'une durée de trois ans pour 180 crédits ECTS, elle sera lancée en août 2018 et servira de base pour la récolte de données de notre recherche postdoctorale ${ }^{1}$. Le Cursus bilingue est commun à deux institutions de formation initiale au degré primaire, l'une francophone, la Haute École Pédagogique des cantons de Berne, Jura et Neuchâtel (HEPBEJUNE) et l'autre germanophone, la Pädagogische Hochschule Bern (PHBern) en Suisse. Précisons ici que les deux institutions se trouvent à la frontière linguistique et que les étudiants suivront leur formation en milieu francophone et germanophone (trois semestres respectifs).

Trois facteurs ont amené à la création progressive de ce projet de partenariat visant à opérationnaliser un dispositif de formation commun entièrement bilingue. Le premier facteur est lié aux changements de la population scolaire helvétique avec des profils d'élèves variés et de cultures différentes. En réponse à une réalité éducative soumise à d'incessantes mutations sociologiques et technologiques, une refonte de la formation initiale des enseignants apparaît comme inéluctable. Profitant de la situation géographique privilégiée des espaces francophones HEP-BEJUNE et germanophones PHBern, au carrefour des deux grandes langues nationales (dans un pays quadrilingue), ce nouveau curriculum professionnalisant (Rectorat et conseil de direction de la HEP-BEJUNE, 2005) permet aux étudiants 


\section{CHRONIQUE}

de s'entraîner pour une réalité omniprésente pour les enseignants, notamment celle du changement permanent qui exige de ces derniers de pouvoir s'adapter constamment.

Dans l'optique d'un reprofilage des enseignants, le deuxième facteur concerne la phase d'accréditation institutionnelle dans laquelle se trouvent les HEP. Avec la nouvelle Loi fédérale sur l'encouragement des hautes écoles et la coordination dans le domaine suisse des hautes écoles du 30 septembre 2011, la Suisse s'est dotée d'un instrument visant à contrôler l'accès à son paysage des hautes écoles et la qualité en soumettant également les Hautes Écoles Pédagogiques à l'accréditation institutionnelle, soit l'une des conditions à remplir pour bénéficier des contributions fédérales. En qualité de projet fortement novateur, le Cursus bilingue stimulera l'innovation dans les deux HEP et permet de porter un regard critique sur le profil de sortie de la formation (les compétences attendues des futurs enseignants), tout en respectant les exigences de la professionnalisation de l'enseignement « entendue comme processus de construction d'une base de connaissances et un répertoire de compétences spécifiques à l'acte d'enseigner » (Lessard, 2017, p. 152). L'intérêt pratique du Cursus bilingue réside donc également dans la capacité à informer les actrices et les acteurs du champ de la formation au sujet des dispositifs de formation monolingues en place, tout en procurant des pistes pour les perfectionner après des analyses approfondies permettant de conceptualiser le curriculum bilingue : un enjeu particulièrement important au moment de l'accréditation institutionnelle.

Le troisième facteur est relatif aux débats actuels autour du sujet de l'enseignement des langues en Suisse qui nous donnent à voir un éventail très large d'arguments pour ou contre l'enseignement d'une deuxième langue nationale au degré primaire. Les argumentations et fondements du débat sont très variés. D'un côté, certains observateurs s'accordent à dire que ce qui compte en réalité, c'est la maîtrise d'une deuxième langue nationale en fin de scolarité obligatoire. D'un autre côté, l'argumentation consiste à insister sur la cohésion nationale. Le plurilinguisme institutionnel peut être renforcé par la promotion de l'enseignement d'une deuxième langue nationale à l'école primaire. Au travers de la rencontre et du respect mutuel des deux communautés linguistiques, le Cursus bilingue travaille également au rapport entre les deux régions linguistiques.

\section{Un concept se basant sur l'enseignement par immersion réciproque}

Le Cursus bilingue permet aux étudiants d'acquérir de bonnes compétences en français et en allemand, mais aussi de s'immerger dans la culture professionnelle de l'autre région linguistique. Son concept repose sur l'enseignement par immersion réciproque (Buser, 2014; Lindholm-Leary et Hargett, 2007), c'est-à-dire que le programme s'efforce de promouvoir le bilinguisme ainsi que l'alphabétisation dans les deux langues d'enseignement en plus de la réussite des études pour tous les étudiants (Christian, 1994). L'enseignement sera diffusé pour moitié en français (HEP-BEJUNE) et pour moitié en allemand (PHBern) avec des classes se composant d'étudiants francophones et germanophones. La mise en contact encadrée, contextualisée et conceptualisée des étudiants avec les pratiques professionnelles des deux régions linguistiques, est tout autant au cœur de la conception du Cursus bilingue que les langues en elles-mêmes. 


\section{Les défis futurs}

Le défi majeur dans cette collaboration n'est pas la langue, mais les différences de conceptions institutionnelles. C'est en pariant sur la volonté commune de voir aboutir le projet, sur le dialogue et le respect mutuel que nous avons trouvé des solutions, par exemple pour lier les deux cadres juridiques.

Soulignons ici que certains étudiants pourront éventuellement vivre un conflit culturel - peut-être renforcé dans une formation bilingue - avec leur culture et les valeurs vécues en famille. Il est parfois difficile de trouver une explication à certains malentendus. Sont-ils dus aux constellations linguistiques ou culturelles ou plutôt personnelles? Souvent, la tendance incite à les expliquer via des différences culturelles précipitées sans analyser les problèmes de façon approfondie comme on le voit également chez des élèves des écoles reposant sur le concept de l'enseignement par immersion réciproque comme la Filière Bilingue (FiBi) à Biel/Bienne (Buser, 2014). Là encore, nous sommes convaincus que grâce au respect mutuel entre les deux communautés linguistiques, nous allons surmonter ces difficultés.

\section{Références}

Buser, M. (2014). Deux plans d'études, une école : scolarisation plurilingue dans la FiBi de Biel/Bienne. Une école publique basée sur le concept de l'enseignement par immersion réciproque. Portrait de la Filière Bilingue. Enjeux pédagogiques,

(23), 31-36.

Christian, D. (1994). Two-way bilingual education: Students learning through two languages. Repéré à https://www.highlineschools.org/cms/lib/WA01919413/Centricity/Domain/86/TW Bilingual Education learning through two languages.pdf

Lessard, C. (2017). Formation initiale et formation continue. La formation des maîtres au Québec. Administration E̋ Éducation, (154), 151-156.

Lindholm-Leary, K. et Hargett, G. (2007). Evaluator's toolkit for dual language programs. Repéré à http://www.cal.org/twi/guidingprinciples.htm

Rectorat et conseil de direction de la HEP-BEJUNE. (2005). Les parcours de formation à la HEP-BEJUNE - Vision et principes curriculaires. Repéré à http://qmpilot.hep-bejune.ch/qmp2/servlet/readObject/?oid=519

\section{Note}

1 Notre recherche postdoctorale sous forme de suivi scientifique s’intitule : «Les savoirs professionnels à la base du "Cursus bilingue/Bilingualer Studiengang" (HEP-BEJUNE \& PHBern) : une étude comparative des deux modèles HEP / PH de professionnalisation et de leurs enjeux en vue de la conceptualisation d'un cursus bilingue commun reposant sur le concept de l'enseignement par immersion réciproque " (sous la direction des Prs Maurice Tardif et Bernard Wentzel).

\section{Pour citer cet article}

Buser, M. (2017). Vers un nouveau profil des enseignants et des enseignantes au primaire en Suisse : bref portrait d'un futur dispositif de formation initiale entièrement bilingue. Formation et profession, 25(3), 113-115. http://dx.doi.org/10.18162/fp.2017.a132 